\title{
THE SURVIVAL OF DARK MATTER HALOS IN THE CLUSTER Cl 0024+16
}

\author{
Priyamvada Natarajan ${ }^{1,2,3}$, Jean-Paul Kneib ${ }^{4}$, Ian Smail $^{5}$, Tommaso Treu $^{6}$, Richard Ellis $^{7,8}$, Sean Moran ${ }^{9}$, \\ Marceau Limousin ${ }^{10}$, ANd Oliver Czoske ${ }^{11}$ \\ ${ }^{1}$ Radcliffe Institute for Advanced Study, Harvard University, 10 Garden Street, Cambridge, MA 02138, USA \\ 2 Department of Astronomy, Yale University, P.O. Box 208101, New Haven, CT 06520-8101, USA \\ ${ }^{3}$ Department of Physics, Yale University, P.O. Box 208120, New Haven, CT 06520-8120, USA \\ ${ }^{4}$ Observatoire Astronomique De Marseille Provence, Marseille, France \\ ${ }^{5}$ Institute for Computational Cosmology, Durham University, South Road, Durham DH1 3LE, UK \\ ${ }^{6}$ Department of Physics, University of California, Santa Barbara, CA 93106-9530, USA \\ ${ }^{7}$ Department of Astronomy, Caltech, 1200 East California Blvd., Pasadena, CA 91125, USA \\ ${ }^{8}$ Department of Astrophysics, Oxford University, Keble Road, Oxford OX1 3RH, UK \\ ${ }^{9}$ Department of Physics \& Astronomy, Johns Hopkins University, 366 Bloomberg Center, 3400 N. Charles Street, Baltimore, MD 21218, USA \\ ${ }^{10}$ Laboratoire d'Astrophysique de Toulouse et Tarbes, Tarbes, France \\ ${ }^{11}$ Kapteyn Astronomical Institute, P.O. Box 800, 9700 AV Groningen, The Netherlands \\ Received 2007 November 27; accepted 2008 November 4; published 2009 March 3
}

\begin{abstract}
Theories of structure formation in a cold dark matter dominated universe predict that massive clusters of galaxies assemble from the hierarchical merging of lower mass subhalos. Exploiting strong and weak gravitational lensing signals inferred from panoramic Hubble Space Telescope imaging data, we present a high-resolution reconstruction of the mass distribution in the massive, lensing cluster $\mathrm{Cl} 0024+16$ at $z=0.39$. Applying galaxy-galaxy lensing techniques we track the fate of dark matter subhalos as a function of projected cluster-centric radius out to $5 \mathrm{Mpc}$, well beyond the virial radius. We report the first detection of the statistical lensing signal of dark matter subhalos associated with late-type galaxies in clusters. The mass of a fiducial dark matter halo that hosts an early-type $L^{*}$ galaxy varies from $M=6.3_{-2.0}^{+2.7} \times 10^{11} M_{\odot}$ within $r<0.6 \mathrm{Mpc}, 1.3_{-0.6}^{+0.8} \times 10^{12} M_{\odot}$ within $r<2.9 \mathrm{Mpc}$, and increases further to $M=3.7_{-1.1}^{+1.4} \times 10^{12} M_{\odot}$ in the outskirts. The mass of a typical dark matter subhalo that hosts an $L^{*}$ galaxy increases with projected cluster-centric radius in line with expectations from the tidal stripping hypothesis. The mass of a dark matter subhalo that hosts a late-type $L^{*}$ galaxy is $1.06_{-0.41}^{+0.52} \times 10^{12} M_{\odot}$. Early-type galaxies appear to be hosted on average in more massive dark matter subhalos compared to late-type galaxies. Early-type galaxies also trace the overall mass distribution of the cluster whereas late-type galaxies are biased tracers. We interpret our findings as evidence for the active assembly of mass via tidal stripping in galaxy clusters. The mass function of dark matter subhalos as a function of projected cluster-centric radius is compared with an equivalent mass function derived from clusters in the Millennium Run simulation populated with galaxies using semianalytic models. The shape of the observationally determined mass functions based on an I-band-selected sample of cluster members and lensing data are in agreement with the shapes of the subhalo mass functions derived from the Millennium Run simulation. However, simulated subhalos appear to be more efficiently stripped than lensing observations suggest. This is likely an artifact of comparison with a dark matter only simulation. Future simulations that simultaneously follow the detailed evolution of the baryonic component during cluster assembly will be needed for a more detailed comparison.
\end{abstract}

Key words: dark matter - galaxies: clusters: general - galaxies: halos

Online-only material: color figure

\section{INTRODUCTION}

Clusters of galaxies are the most massive and recently assembled structures in the universe. In the context of the hierarchical growth of structure in a cold dark matter (CDM) dominated universe, clusters are the repository of copious amounts of the dark matter. Gravitational lensing, predicted by Einstein's theory of general relativity, is the deflection of light rays from distant sources by foreground mass structures. In its most dramatic manifestation, strong lensing requires a rare alignment with foreground dense structures and produces highly distorted, magnified, and multiple images of a single background source (Schneider et al. 1992). More commonly, the observed shapes of background sources viewed via a foreground cluster lens are systematically elongated, in the so-called weak lensing regime. Strong and weak lensing offer the most reliable probes of the distribution of dark matter on various cosmic scales (Blandford \& Narayan 1992; Mellier 2002; Schneider et al. 1992).
Strong lensing studies of the core regions of several clusters indicate that the dark matter distribution can be represented by a combination of smoothly distributed, extended cluster mass components and smaller-scale clumps or subhalos associated with luminous galaxies (Kneib et al. 1996; Natarajan \& Kneib 1997; Natarajan et al. 1998). The smooth components have been detected using weak lensing techniques out to the turnaround radius (typically of the order of several Mpc) in clusters (Kneib et al. 2003; Gavazzi et al. 2003; Broadhurst et al. 2005; Bradac et al. 2006; Clowe et al. 2006; Wittman et al. 2006; Limousin et al. 2009; Bardeau et al. 2007). To date, however, attention has largely focused on the lensing-derived density profile of the smooth cluster component, and its agreement with profiles computed from high-resolution numerical simulations of structure formation in the universe (Navarro et al. 1997; Navarro et al. 2004; Sand et al. 2004). In fact, the granularity of the dark matter distribution associated with individual galactic subhalos holds important clues to the growth and assembly of 
clusters. Several earlier studies have explored this issue for the particular case of $\mathrm{Cl}$ 0024+16 (Tyson et al. 1998; Broadhurst et al. 2000; Smail et al. 1996).

The detailed mass distribution of clusters, and in particular the fraction of the total cluster mass associated with individual galaxies, has important consequences for the frequency and nature of galaxy interactions in clusters (Merritt 1983; Moore et al. 1996; Ghigna et al. 1998; Okamoto \& Habe 1999; Abadi et al. 1999). Infalling subhalos suffer a range of violent fates as the strong gravitational potential of the cluster tidally strips dark matter and removes baryons via ram-pressure stripping from them (Cortese et al. 2008). Simulations suggest that subhalos may not be arranged equally around galaxies of different morphologies given their varying histories in the cluster environment (Ghigna et al. 1998; Tormen et al. 1998; Springel et al. 2001). Moreover, subhalos may become tidally truncated by an amount that will differ substantially over the large dynamic range in cluster density. Observations of tidal stripping offer important clues to key questions regarding the growth and evolution of clusters. How much dark matter is associated with the subhalos in clusters as a function of radius? To what extent do the luminous cluster galaxy populations trace the detailed mass distribution? And, how significant is tidal stripping for the various morphological galaxy types in the cluster? These are questions we attempt to answer in this work using observational data and by comparing with numerical simulations.

To explore cluster galaxy masses, we exploit the technique of galaxy-galaxy lensing, which was originally proposed as a method to constrain the masses and spatial extents of field galaxies (Brainerd et al. 1996; Schneider \& Rix 1997), which has been since extended and developed over the years to apply inside clusters (Natarajan \& Kneib 1996; Geiger \& Schneider 1998; Natarajan et al. 1998, 2002, 2007; Limousin et al. 2007a). Previous attempts to measure the granularity of the dark matter distribution as a function of cluster-centric radius from observations have had limited success. Analyzing Canada-FranceHawaii Telescope (CFHT) weak lensing data from the supercluster MS0302+17, Gavazzi et al. (2004) claimed detection of a radial trend in the extents of dark matter subhalos in this supercluster region extending out to a few Mpc from the center. Gavazzi et al. reported that the mass distribution derived from weak lensing was robustly traced by the luminosity of earlytype galaxies, although their analysis did not include late-type galaxies or a large-scale smooth component. However, utilizing ground-based CFHT weak lensing data for a sample of massive clusters at $z=0.2$, Limousin et al. (2005) did not detect any variation of the dark matter subhalo masses with cluster-centric radius out to a significant fraction of the virial radius. The resolution of ground-based data appears to be inadequate to detect this effect.

In this paper, we present the determination of the mass function of substructure in $\mathrm{Cl} 0024+16$ (at $z=0.39$ ) in three radial bins using panoramic Hubble Space Telescope (HST) imaging data. A high-resolution mass model tightly constrained by current observations is constructed including individual cluster galaxies and their associated dark matter subhalos. We show that over a limited mass range we can successfully construct the mass function of subhalos inside this cluster as a function of cluster-centric radius. The three bins span from the center to $5 \mathrm{Mpc}$ (well beyond the virial radius of $1.7 \mathrm{Mpc}$ ) providing us insights into the tidal stripping process. We also compare properties of the subhalos that host early-type galaxies with those that host late-type galaxies in $\mathrm{Cl} 0024+16$. In addition, we com- pare the results retrieved from the lensing analysis with results from the largest cosmological simulation carried out so farthe Millennium Simulation. $N$-body simulations in combination with the semianalytic models that we employ in this work are an invaluable tool for investigating the nonlinear growth of structure in detail and to provide insights into the cluster assembly process.

The outline of this paper is as follows: in Section 2, we discuss the theoretical framework of tidal stripping and galaxy-galaxy lensing in clusters; in Section 3, the observations and modeling are described. The analysis for $\mathrm{Cl} 0024+16$ is presented in Section 4 including a discussion of the uncertainties; results and the comparison with clusters in the Millennium Simulation are described in Section 5. We conclude with a discussion of the implications of our results for the LCDM model and the future prospects of this work. ${ }^{12}$

\section{THEORETICAL FRAMEWORK}

\subsection{Tidal Stripping and Dynamical Modification in Clusters}

Theoretical studies of cluster formation using simulations and analytic models predict that there are two key dynamical processes (Ghigna et al. 1998; Springel et al. 2001; De Lucia et al. 2004; Moore et al. 1996; Balogh et al. 2000; Merritt 1985) that are relevant to the mass loss of infalling dark matter subhalos in assembling clusters. The first process is tidal stripping induced by the interaction of infalling galaxies and groups with the global tidal field generated by the smooth dark matter distribution. The second process is modification to the mass distribution due to high- and lowvelocity encounters between infalling subhalos (Moore et al. 1996).

For the purposes of studying the dynamics of galaxies in clusters we have partitioned the cluster into three distinct regions: the inner core region where the global tidal field is the strongest, the transition region where the two above-mentioned dynamically transformative processes occur, and finally, the periphery where the dominant stripping is due to interactions between the infalling galaxies and groups rather than the global tidal field (Treu et al. 2003). A detailed study of the properties of cluster galaxies in $\mathrm{Cl} 0024+16$ by Treu et al. 2003 finds that demarcation into these three regions is naturally provided by the dynamical processes that operate efficiently at various radii from the cluster center.

In the central region, the gravitational potential of the cluster is the strongest and tidal stripping is expected to be the dominant dynamically transformative process. Recent tidal effects are not expected in the transition region whereas most galaxies inhabiting the periphery are likely to have never traversed the cluster center. The galaxies in the outer regions are expected to be modified predominantly due to local interactions with other nearby galaxies and groups despite being gravitationally bound to the cluster.

An analytic estimate of the effect of tidal truncation as a function of cluster-centric radius can be calculated by modeling $\mathrm{Cl} \mathrm{0024+16} \mathrm{as} \mathrm{an} \mathrm{isothermal} \mathrm{mass} \mathrm{distribution} \mathrm{and} \mathrm{considering}$ the motions of cluster subhalos in this potential (Merritt 1985). In this framework, the tidal radius of a subhalo hosting a cluster

\footnotetext{
12 Throughout this work wherever required we have used the following values for the cosmological parameters: $H_{0}=72 \mathrm{~km} \mathrm{~s}^{-1} \mathrm{Mpc}^{-1} ; \Omega_{\mathrm{m}}=0.3 ; \Omega_{\Lambda}=$ 0.7 . At the redshift of $\mathrm{Cl} 0024+16,1^{\prime \prime}=5.184 \mathrm{kpc}$.
} 
galaxy is given by

$$
R_{\text {tidal }} \propto\left(\frac{\sigma_{\text {gal }}}{\sigma_{\text {cluster }}}\right) r,
$$

where $R_{\text {tidal }}$ is the tidal radius of the subhalo, $\sigma_{\text {gal }}$ is the central velocity dispersion of the galaxy, $\sigma_{\text {cluster }}$ is the velocity dispersion of the cluster, and $r$ is the distance from the cluster center. The current paradigm for structure formation in the universe predicts that the masses of infalling subhalos are a strong function of cluster-centric radius $r$, indicative of the variation of the strength of tidal stripping from the periphery (where it is modest) to the inner regions, where it is severe (Springel et al. 2001; De Lucia et al. 2004; Moore et al. 1996; Balogh et al. 2000). Mapping the mass function of subhalos directly from observations offers a powerful way to test these theoretical predictions.

\subsection{Galaxy-Galaxy Lensing in Clusters}

In this subsection, we briefly outline the analysis framework. Details can be found in several earlier papers (Natarajan \& Kneib 1996; Natarajan et al. 1998, 2002, 2007). For the purpose of constraining the properties of the subhalo population, $\mathrm{Cl} 0024+16$ is modeled parametrically as a super-position of smooth large-scale mass components, which we will refer to with subscript "s" hereafter, and smaller scale potentials that are associated with bright cluster members, referred to as perturbers denoted by the subscript " $p_{i}$." Using the same data set to construct a mass distribution for $\mathrm{Cl} 0024+16$ Kneib et al. (2003) found that the best-fit model required two largescale components. In our current modeling, we adopt that parameterization as the prior. In earlier work, our analysis was limited by data to the inner regions of clusters $(<1 \mathrm{Mpc})$, and only to early-type galaxies as perturbers as a consequence (Natarajan et al. 1998, 2002, 2007). With the current data set we also probe the late-type cluster member population and statistically constrain parameters that characterize their dark matter subhalos. There are, however, an insufficient number of late-types in the core region, their numbers steadily increase with cluster-centric radius. Therefore, in the core region, we focus on the subhalos of early types. In effect, the contribution of late types in the core region gets inevitably taken into account as part of the smooth mass distributions. We note here that while we illustrate our formalism with simple equations to provide insight into our framework; ultimately, the analysis is performed numerically and all the nonlinearities arising in the lensing inversion are taken into account. The gravitational potential of $\mathrm{Cl} 0024+16$ is modeled as follows:

$$
\phi_{\mathrm{tot}}=\Sigma_{n} \phi_{\mathrm{s}}+\Sigma_{i} \phi_{\mathrm{p}_{\mathrm{i}}}
$$

where the two $\phi_{\mathrm{s}}(n=1$ and $n=2)$ components represent the potentials that characterize the smooth component and $\phi_{\mathrm{p}_{\mathrm{i}}}$ are the potentials of the galaxy subhalos treated as perturbers. The corresponding deflection angle $\alpha_{I}$ and the amplification matrix $A^{-1}$ can also be decomposed into independent contributions from the smooth clumps and perturbers,

$$
\begin{gathered}
\alpha_{I}=\Sigma_{n} \nabla \phi_{\mathrm{s}}+\Sigma_{i} \nabla \phi_{\mathrm{p}_{\mathrm{i}}}, \\
A^{-1}=I-\Sigma_{n} \nabla \nabla \phi_{\mathrm{s}}-\Sigma_{i} \nabla \nabla \phi_{\mathrm{p}_{\mathrm{i}}} .
\end{gathered}
$$

In fact, the amplification matrix can be decomposed as a linear sum:

$$
A^{-1}=\left(1-\Sigma_{n} \kappa_{\mathrm{s}}-\Sigma_{i} \kappa_{\mathrm{p}}\right) I-\Sigma_{n} \gamma_{\mathrm{s}} J_{2 \theta_{\mathrm{s}}}-\Sigma_{i} \gamma_{\mathrm{p}_{\mathrm{i}}} J_{2 \theta_{\mathrm{pi}}},
$$

where $\kappa$ is the magnification and $\gamma$ the shear. The shear $\gamma$ is written as a complex number and is used to define the reduced shear $\bar{g}$, which is the quantity that is measured directly from observations of the shapes of background galaxies. The reduced shear can also be further decomposed into contributions from the smooth pieces and the perturbers

$$
\overline{g_{\text {tot }}}=\frac{\bar{\gamma}}{1-\kappa}=\frac{\Sigma_{n} \bar{\gamma}_{\mathrm{s}}+\Sigma_{i} \bar{\gamma}_{p_{i}}}{1-\Sigma_{n} \kappa_{\mathrm{s}}-\Sigma_{i} \kappa_{p_{i}}} .
$$

Here $\bar{\gamma}$ is the mean shear of background galaxies in an annulus around a particular early-type cluster galaxy treated as a local perturber. In the frame of an individual perturber $j$ (neglecting effect of perturber $i$ if $i \neq j$ ), the above simplifies to

$$
\left.\bar{g}_{\text {tot }}\right|_{j}=\frac{\sum_{n} \bar{\gamma}_{s} \bar{\gamma}_{p j}}{1-\sum_{n} \kappa_{s}-\kappa_{p j}} .
$$

Restricting our analysis to the weak regime (as mentioned above the analysis is ultimately performed numerically and includes the effect of strong lensing), and thereby retaining only the firstorder terms from the lensing equation for the shape parameters (e.g., Kneib et al. 1996) we have

$$
\bar{g}_{I}=\bar{g}_{S}+\bar{g}_{\text {tot }},
$$

where $\bar{g}_{I}$ is the distortion of the image, $\bar{g}_{S}$ the intrinsic shape of the source, $\bar{g}_{\text {tot }}$ is the distortion induced by the lensing potentials (the smooth component as well as the perturbers). Note that the equations are outlined here to provide a feel for the technique. The lensing inversion for the observational data is done numerically taking the full nonlinearities that rise in the strong lensing regime into account. ${ }^{13}$

In the local frame of reference of the subhalos, the mean value of the quantity $\bar{g}_{I}$ and its dispersion are computed in circular annuli (at radius $r$ from the perturber center), assuming a known value for the smooth cluster component over the area of integration. In the frame of the perturber, the averaging procedure allows efficient subtraction of the large-scale component, enabling the extraction of the shear component induced in the background galaxies only by the local perturber. The background galaxies are assumed to have intrinsic ellipticities drawn from a known distribution (see the Appendix for further details). Schematically, the effect of the cluster on the intrinsic ellipticity distribution of background sources is to cause a coherent displacement and the presence of perturbers merely adds small-scale noise to the observed ellipticity distribution. Since we are subtracting a long-range signal to statistically extract a smaller scale anisotropy riding on it, we are inherently limited to physical scales on which the contrast is maximal, i.e., galaxy subhalo scales.

The contribution of the smooth cluster component has two effects: it boosts the shear induced by the perturber which becomes nonnegligible in the cluster center, and it simultaneously dilutes the regular galaxy-galaxy lensing signal due to the $\sigma_{\bar{g}_{s}}^{2} / 2$ term in the dispersion. However, one can in principle optimize the noise by "subtracting" the measured cluster signal $\bar{g}_{\mathrm{s}}$ using a tightly constrained parametric model for the cluster.

The feasibility of this differenced averaging prescription for extracting the distortions induced by the possible presence

\footnotetext{
13 The measured image shape and orientation are used to construct a complex number whose magnitude is given in terms of the semimajor axis (1) and semiminor axis (2) of the image and the orientation is the phase of the complex number.
} 
of dark matter subhalos around cluster galaxies with HST quality data has been amply demonstrated in our earlier papers (Natarajan et al. 1998, 2002, 2004, 2007). We have also shown with direct comparison to simulations that we can reliably recover substructure mass functions with this technique in the inner $1 \mathrm{Mpc}$ or so of galaxy clusters. Note here that it is the presence of the underlying large-scale smooth mass components (with a high value of $\kappa_{S}$ ) that enables the extraction of the weaker signal riding on it.

\section{OBSERVATIONS AND MODELING}

\subsection{The HST WFPC-2 Data Set}

Our data set comprises a mosaic of 39 sparsely sampled images of the rich cluster $\mathrm{Cl} 0024+16(z=0.39)$ taken by the Wide Field Planetary Camera-2 on the HST. By applying lensing techniques to this panoramic imaging data set, we aim to characterize the fine scale distribution of dark matter. This unique data set extends to the turnaround radius $\simeq 5 \mathrm{Mpc}$, well beyond the inner $0.5-1 \mathrm{Mpc}$ that has been studied previously. This enables us to map the detailed dark matter distribution and to calibrate the tidal stripping effect as a function of distance from the cluster center. In an earlier analysis, we combined strong and weak lensing constraints to provide an accurate representation of the smooth dark matter component out to $5 \mathrm{Mpc}$ radius (Kneib et al. 2003). Strong lensing provides stringent constraints on the mass profile in the inner region while the detected weak shear constrains the profile out at large radii (Mellier 2002; Kneib et al. 2003). Noncontiguous, sparse sampling of the HST pointings was chosen to maximize radial coverage. Further details of the data and analyses can be found in earlier published papers (Kneib et al. 2003; Treu et al. 2003). We reiterate here that the WFPC-2 data set used for this analysis has been presented already and is described in detailed in earlier works by our group, including the determination of shapes for the background galaxies (Kneib et al. 2003); selection and confirmation of cluster membership and morphological classifications of cluster galaxies (Treu et al. 2003).

Here, we use galaxy-galaxy lensing to detect cluster galaxy subhalos associated with early- and late-type galaxies against the background of smoothly distributed dark matter in three radial bins. Using the extensive set of ground-based spectra (Czoske et al. 2001, 2002; Moran et al. 2005) and HST morphologies (Treu et al. 2003), we first identified early- and late-type members to well beyond the virial radius, $\left(r_{\mathrm{vir}}=1.7 \mathrm{Mpc}\right)$, out to $\sim 5 \mathrm{Mpc}$. Details of the data reduction, cluster membership determination, and morphological classification can be found in Treu et al. (2003).

\subsection{Modeling the Cluster $\mathrm{Cl} 0024+16$}

$\mathrm{Cl} \mathrm{0024+16} \mathrm{is} \mathrm{an} \mathrm{extremely} \mathrm{massive} \mathrm{cluster} \mathrm{and} \mathrm{has} \mathrm{a} \mathrm{surface}$ mass density in the inner regions which is significantly higher than the critical value, therefore produces a number of multiple images of background sources. By definition, the critical surface mass density for strong lensing is given by

$$
\Sigma_{\text {crit }}=\frac{c^{2}}{4 \pi G} \frac{D_{s}}{D_{d} D_{d s}},
$$

where $D_{s}$ is the angular diameter distance between the observer and the source, $D_{d}$ is the angular diameter distance between the observer and the deflecting lens, and $D_{d s}$ is the angular diameter distance between the deflector and the source.
Note that the integrated lensing signal detected is due to all the mass distributed along the line of sight in a cylinder projected onto the lens plane. In this and all other cluster lensing work, the assumption is made that individual clusters dominate the lensing signal as the probability of encountering two massive rich clusters along the same line of sight is extremely small due to the fact that these are very rare objects in hierarchical structure formation models. $\mathrm{Cl} 0024+16$ is known to have a significant amount of substructure in velocity space. Czoske et al. (2002) and more recently Moran et al. (2005) have performed comprehensive redshift surveys of this cluster and its environs and have enabled the construction of a threedimensional picture for this cluster using the $\sim 500$ galaxy redshifts within about 3-5 Mpc from the cluster center. Their combined data reveal a foreground component of galaxies separated from the main cluster in velocity space. Both groups argue that this is likely a remnant of a high-speed collision between the main cluster and an infalling subcluster. The detailed redshift distribution of cluster members in $\mathrm{Cl} 0024+16$ is taken carefully into account in our lensing analysis, starting with a prior that includes two large-scale components to model the smooth mass distribution.

With our current sensitivity limits, galaxy-galaxy lensing within the cluster provides a determination of the total enclosed mass within an aperture. We lack sufficient sensitivity to constrain the detailed mass profile for individual cluster galaxies. With higher-resolution data in the future we hope to be able to obtain constraints on the slopes of mass profiles within subhalos. In this paper, the subhalos are modeled as pseudo-isothermal elliptical components (PIEMD models, derived by Kassiola \& Kovner 1993) centered on galaxies that lie within a projected radius of out to $5 \mathrm{Mpc}$ from the cluster center and two NFW profiles are used to model the smooth, large-scale contribution. We find that the final results obtained for the characteristics of the subhalos (or perturbers) is largely independent of the form of the mass distribution used to model the smooth, largescale components. A comparison of the best-fit profiles for the smooth component from lensing with those obtained in highresolution cosmological $N$-body simulations has been presented in the work of Kneib et al. (2003). Combining strong and weak constraints, they were able to probe the mass profile of the cluster on scales of $0.1-5 \mathrm{Mpc}$, thus providing a valuable test of the universal form proposed by Navarro, Frenk, \& White (NFW) on large scales. We use the best-fit mass model of Kneib et al. (2003) for the smooth component as a prior in our analysis, although we allow the parameters like the centroids of the two large-scale components and their velocity dispersion to vary when obtaining constraints on the subhalos. The two NFW components used as priors are characterized by the following properties-Clump 1: with $M_{200}=6.5 \times 10^{14} M_{\odot} ; c=22_{-5}^{+9} ; r_{200}=1.9 \mathrm{Mpc}$; $r_{s}=88 \mathrm{kpc}$; and Clump 2: with $M_{200}=2.8 \times 10^{14} M_{\odot}$; $c=4_{-1}^{+2} ; r_{200}=1.5 \mathrm{Mpc} ; r_{s}=364 \mathrm{kpc}$.

To quantify the lensing distortion induced, the individual galaxy-scale halos are modeled using the PIEMD profile with

$$
\Sigma(R)=\frac{\Sigma_{0} r_{0}}{1-r_{0} / r_{t}}\left(\frac{1}{\sqrt{r_{0}^{2}+R^{2}}}-\frac{1}{\sqrt{r_{t}^{2}+R^{2}}}\right),
$$

with a model core-radius $r_{0}$ and a truncation radius $r_{t} \gg r_{0}$. Correlating the above mass profile with a typical de Vaucleours light profile (the observed profile for bright early-type galaxies) provides a simple relation between the truncation radius and the 
effective radius $R_{\mathrm{e}}, r_{t} \sim(4 / 3) R_{\mathrm{e}}$. The coordinate $R$ is a function of $x, y$ and the ellipticity

$$
R^{2}=\left(\frac{x^{2}}{(1+\epsilon)^{2}}+\frac{y^{2}}{(1-\epsilon)^{2}}\right) ; \epsilon=\frac{a-b}{a+b} .
$$

The mass enclosed within an aperture radius $R$ for the $\epsilon=0$ model is given by

$$
M(R)=\frac{2 \pi \Sigma_{0} r_{0}}{1-\frac{r_{0}}{r_{t}}}\left[\sqrt{r_{0}^{2}+R^{2}}-\sqrt{r_{t}^{2}+R^{2}}+\left(r_{t}-r_{0}\right)\right] .
$$

The total mass $M$, is finite with $M \propto \Sigma_{0} r_{0} r_{t}$. The shear is

$$
\begin{aligned}
\gamma(R) & =\kappa_{0}\left[-\frac{1}{\sqrt{R^{2}+r_{0}^{2}}}+\frac{2}{R^{2}}\left(\sqrt{R^{2}+r_{0}^{2}}-r_{0}\right)\right. \\
& \left.+\frac{1}{\sqrt{R^{2}+r_{t}^{2}}}-\frac{2}{R^{2}}\left(\sqrt{R^{2}+r_{t}^{2}}-r_{t}\right)\right] .
\end{aligned}
$$

In order to relate the light distribution in cluster galaxies to key parameters of the mass model of subhalos, we adopt a set of physically motivated scaling laws derived from observations (Brainerd et al. 1996; Limousin et al. 2005; Halkola et al. 2007)

$$
\sigma_{0}=\sigma_{0 *}\left(\frac{L}{L^{*}}\right)^{\frac{1}{4}} ; r_{0}=r_{0 *}\left(\frac{L}{L^{*}}\right)^{\frac{1}{2}} ; r_{t}=r_{t *}\left(\frac{L}{L^{*}}\right)^{\alpha} \text {. }
$$

The total mass $M$ enclosed within an aperture $r_{t *}$ and the total mass-to-light ratio $M / L$ then scale with the luminosity as follows for the early-type galaxies

$M_{\text {ap }} \propto \sigma_{0 *}^{2} r_{t *}\left(\frac{L}{L^{*}}\right)^{\frac{1}{2}+\alpha}, \quad M / L \propto \sigma_{0 *}^{2} r_{t *}\left(\frac{L}{L^{*}}\right)^{\alpha-1 / 2}$,

where $\alpha$ tunes the size of the galaxy halo. In this work $\alpha$ is taken to be $1 / 2$. These scaling laws are empirically motivated by the Faber-Jackson relation for early-type galaxies (Brainerd et al. 1996). For late-type cluster members, we use the analogous Tully-Fisher relation to obtain scalings of $\sigma_{0 *}$ and $r_{t *}$ with luminosity. The empirical Tully-Fisher relation has significantly higher scatter than the Faber-Jackson relation. In this analysis, we do not take the scatter into account while employing these scaling relations. We assume these scaling relations and recognize that this could ultimately be a limitation but the evidence at hand supports the fact that mass traces light efficiently both on cluster scales (Kneib et al. 2003) and on galaxy scales (Wilson et al. 2001; Mandelbaum et al. 2006). Further explorations of these scaling relations have recently been presented in Halkola \& Seitz (2007) and Limousin et al. (2007a). The redshift distribution and intrinsic ellipticity distribution assumed for this analysis are outlined in the Appendix.

\subsection{The Maximum-Likelihood Method}

Parameters that characterize both the global components and the subhalos are optimized, using the observed strong lensing features-positions, magnitudes, geometry of multiple images, and measured spectroscopic redshifts, along with the smoothed shear field as constraints. With the parameterization presented above, we optimize and extract values for the central velocity dispersion and the aperture scale $\left(\sigma_{0 *}, r_{t *}\right)$ for a subhalo hosting a fiducial $L^{*}$ cluster galaxy.
Maximum-likelihood analysis is used to obtain significance bounds on these fiducial parameters that characterize a typical $L^{*}$ subhalo in the cluster. The likelihood function of the estimated probability distribution of the source ellipticities is maximized for a set of model parameters, given a functional form of the intrinsic ellipticity distribution measured for faint galaxies. For each "faint" galaxy $j$, with measured shape $\tau_{\mathrm{obs}}$, the intrinsic shape $\tau_{S_{j}}$ is estimated in the weak regime by subtracting the lensing distortion induced by the smooth cluster models and the galaxy subhalos

$$
\tau_{S_{j}}=\tau_{\mathrm{obs}_{\mathrm{j}}}-\Sigma_{i}^{N_{c}} \gamma_{p_{i}}-\Sigma_{n} \gamma_{c},
$$

where $\Sigma_{i}^{N_{c}} \gamma_{p_{i}}$ is the sum of the shear contribution at a given position $j$ from $N_{c}$ perturbers. This entire inversion procedure is performed numerically using the code developed that builds on the ray-tracing routine LENSTOOL written by Kneib (1993). This machinery accurately takes into account the nonlinearities arising in the strong lensing regimes well. Using a welldetermined "strong lensing" model for the inner regions along with the shear field and assuming a known functional form for $p\left(\tau_{S}\right)$ the probability distribution for the intrinsic shape distribution of galaxies in the field, the likelihood for a guessed model is given by

$$
\mathcal{L}\left(\sigma_{0 *}, r_{t *}\right)=\Pi_{j}^{N_{\text {gal }}} p\left(\tau_{S_{j}}\right),
$$

where the marginalization is done over $\left(\sigma_{0 *}, r_{t *}\right)$. We compute $\mathcal{L}$ assigning the median redshift corresponding to the observed source magnitude for each arclet. The best fitting model parameters are then obtained by maximizing the log-likelihood function $l$ with respect to the parameters $\sigma_{0 *}$ and $r_{t *}$. Note that the parameters that characterize the smooth component are also simultaneously optimized. In this work, we perform this likelihood analysis in each of the three radial bins to obtain the set of $\left(\sigma_{0 *}, r_{t *}\right)$ that characterize subhalos in each radial bin.

In summary, the basic steps of our analysis therefore involve lens inversion, modeling, and optimization, which are done using the LENSTOOL software utilities (Kneib 1993; Jullo et al. 2007). These utilities are used to perform the ray tracing from the image plane to the source plane with a specified intervening lens. This is achieved by solving the lens equation iteratively, taking into account the observed strong lensing features, positions, geometry, and magnitudes of the multiple images. In this case, we also include a constraint on the location of the critical line (between two mirror multiple images) to tighten the optimization. We fix the core radius of an $L^{*}$ subhalo to be $0.1 \mathrm{kpc}$, as by construction our analysis cannot constrain this quantity. The measured shear field and the measured velocity dispersions of early-type galaxies are used as priors in the likelihood estimation. In addition to the likelihood contours, the reduced $\chi^{2}$ for the best-fit model is also found to be robust.

\section{ANALYSIS FOR CL 0024+16}

To detect cluster subhalos, we first select a population of background galaxies within a magnitude range $23<I<26$ (measured in the F814W filter) and determined their individual shapes to a high degree of accuracy taking into account the known anisotropy of the point spread function of the WFPC2 Camera (Bridle et al. 2002; Kuijken 1999). Details of this procedure and the systematics are described in detail in Kneib et al. (2003). Shape distortions in this population were then used 


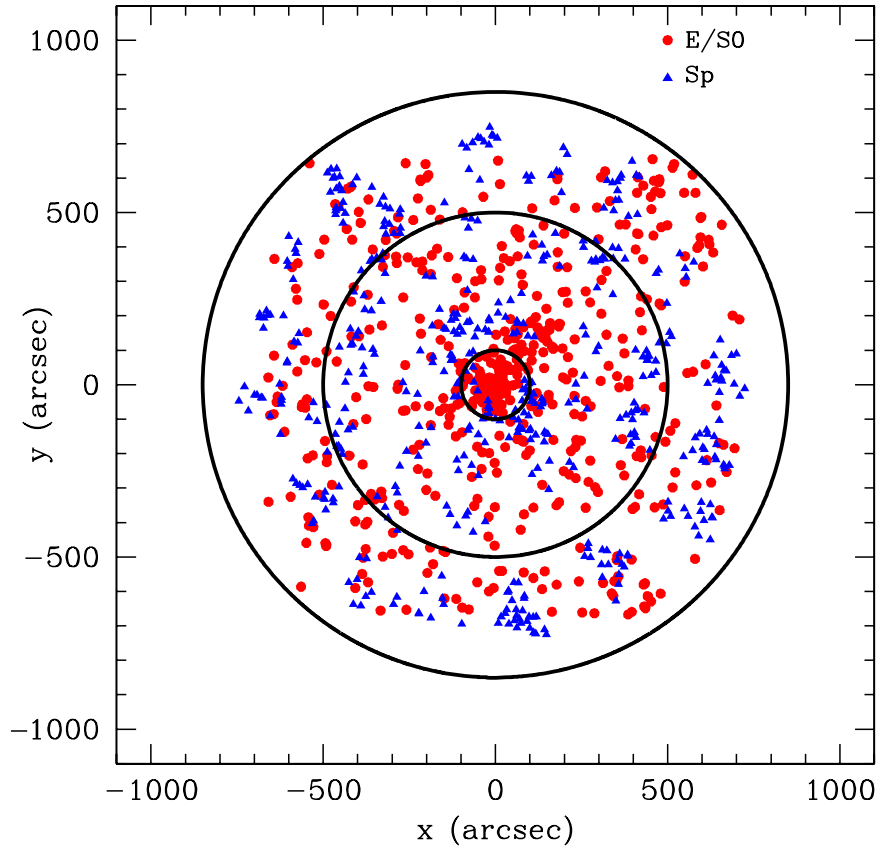

Figure 1. Spatial distribution of morphologically classified early-type galaxies (red circles) and late-type galaxies (blue triangles) with measured redshifts in $\mathrm{Cl} 0024+16$ derived from the sparsely sampled mosaic using the WFPC-2 Camera aboard the HST. The three circles define the radial binning used in our analysis. The innermost circle encompasses the core region of the cluster out to $0.6 \mathrm{Mpc}$, the middle circle the transition region extending out to $2.9 \mathrm{Mpc}$ and the outer circle marks the periphery of the cluster out to $4.8 \mathrm{Mpc}$. Galaxies plotted here include spectroscopically confirmed cluster members and galaxies with secure photometric redshifts in the $H S T$ footprint.

(A color version of this figure is available in the online journal.)

to compute the masses of the foreground cluster and its member subhalos.

To quantify environmental effects on infalling dark matter halos and noting the three physical regimes discussed earlier, we divided the cluster into three regions: the central region extending out to $\sim 600 \mathrm{kpc}$ from the center (core); the transition region extending out to $\sim 1.7 r_{\text {vir }} \sim 2.9 \mathrm{Mpc}$, (transition) and the periphery out to $\sim 2.8 r_{\text {vir }} \sim 4.8 \mathrm{Mpc}$ (periphery). These bins partition the cluster into regions of high, medium, and low galaxy number density and dark matter density (Treu et al. 2003), respectively. The typical surface density of cluster members in the core region is 120 galaxies per $\mathrm{Mpc}^{-2}$; in the transition region it drops to about 60 galaxies per $\mathrm{Mpc}^{-2}$ and in the periphery it is roughly 50 galaxies per $\mathrm{Mpc}^{-2}$ (Treu et al. 2003).

A well-defined morphology-density relation is detected in Cl 0024+16 (Treu et al. 2003; Dressler 1980; Fasano et al. 2000). The fraction of early-type galaxies declines steeply away from the center, starting at $70 \%-80 \%$ out to $1 \mathrm{Mpc}$ and decreasing down to $50 \%$ at the outskirts. In contrast, the late-type galaxy population fraction is negligible in the center but increases in the transition region and constitutes $50 \%$ out at the periphery. In fact, Moran et al. (2007) find that the spirals are kinematically disturbed even well beyond the virial radius in this cluster. In the core, cluster membership was defined strictly, and only spectroscopically confirmed members were used in the galaxy-galaxy lensing analysis. In the transition region and the periphery, the classification of cluster members was performed using both spectroscopically and photometrically determined redshifts. We selected cluster

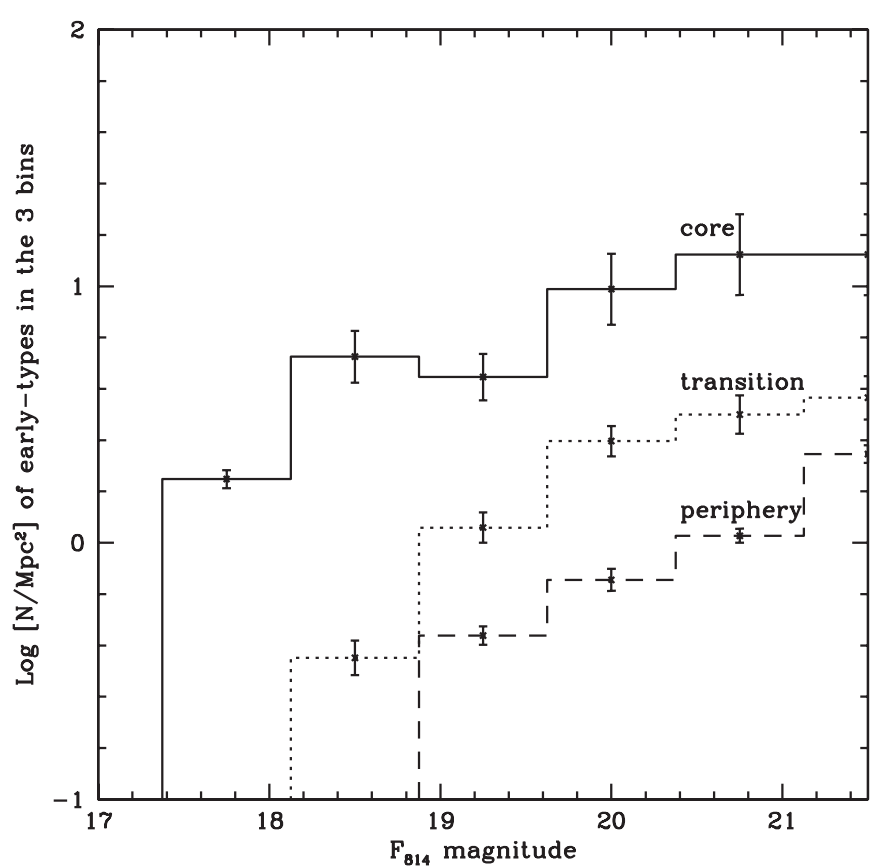

Figure 2. Luminosity function of early-type galaxies in the three regions: the number of galaxies per unit area versus magnitude is shown. It is clear from this plot that there is no systematic luminosity selection bias with cluster-centric radius for the early-type cluster members. However, luminosity segregation is evident in the core region. The luminosity function plotted above includes spectroscopically confirmed cluster members and those with secure photometric redshifts in the $H S T$ footprint.

galaxies within $17<I<22$ to ensure comparable degrees of completeness for both morphological types across all three bins. Our selection procedure yields 51 early types in the core; 93 in the transition region (70 spectroscopically confirmed) and 44 (15 spectroscopically confirmed) in the periphery. Including early types from the ground based survey work (Moran et al. 2007), we have an additional 257 members in the transition region and 294 members in the periphery. There are a total of 331 late types (this inventory includes the HST mosaic and ground-based data) confined to transition and periphery region. For the early types in the HST WFPC-2 mosaic, all 51 in the core are spectroscopically confirmed to be cluster members, in the outer two bins, about $63 \% \pm 7 \%$ of the early types are spectroscopically confirmed, and across all morphologies $\sim 65 \%$ have secure measured redshifts. In addition, we have redshifts for early-type candidates that lie outside our tiled HST mosaic as well photometric redshifts estimates. The radial distribution of the selected cluster galaxies is shown in Figure 1. The similarity of the luminosity function of the selected early types in the three bins shown in Figure 2 ensures that we have truly comparable samples with no luminosity bias.

For each radial bin and type, we applied the likelihood analysis described above to extract the best-fit parameters and significance bounds for the dark matter halo associated with a fiducial $L^{*}$ subhalo in the cluster. Gravitational lensing effects are sensitive to the total mass $M$ enclosed by a subhalo within an aperture $r_{t *}$. To account for the differing mass-tolight ratios of the early and late-type galaxies, we utilized the well-known empirical relations between the velocity dispersion and luminosity for early types (Faber-Jackson relation); and equivalently that between the circular velocity and luminosity (Tully-Fisher relation) for late-type galaxies. We used the relations determined for $\mathrm{Cl} 0024+16$ by Moran et al. (2005, 
2007) to relate mass and light in our modeling procedure. An $L^{*}$ early-type galaxy and a late-type galaxy are assumed to have the same luminosity. The limitations and systematics of the galaxy-galaxy lensing analysis in clusters have been described in detail in our all our earlier papers, below we briefly mention some of the key uncertainties of this method.

The following basic tests were performed for $\mathrm{Cl} 0024+16$, (1) choosing random locations (instead of bright, cluster member locations) for the perturbers; (2) scrambling the shapes of background galaxies; and (3) choosing to associate the perturbers with the faintest (as opposed to the brightest) galaxies. None of the above yield a convergent likelihood map, in fact all that is seen in the resultant two-dimensional likelihood surfaces is noise for all the above test cases.

While the robustness of our method has been extensively tested and reported in detail in earlier papers, there are a couple of caveats and uncertainties inherent to the technique that ought to be mentioned. In galaxy-galaxy lensing, we are only sensitive to a restricted mass range in terms of secure detection of substructure. This is due to the fact that we are quantifying a differential signal above the average tangential shear induced by the smooth cluster component. Therefore, we are inherently limited by the average number of distorted background galaxies that lie within the aperture scale radii of cluster galaxies. This trade-off between requiring a sufficient number of lensed background galaxies in the vicinity of the subhalos and the optimum locations for the subhalos leads us to choose the brightest cluster galaxies in each radial bin. It is possible that the bulk of the mass in subhalos is in lower mass clumps, which in this analysis is essentially accounted for as being part of the smooth components. Also we cannot sensibly quantify the contribution of close pairs/neighbors individually as it is essentially a statistical technique.

Our results are robust and we statistically determine the mass of a dark matter subhalo that hosts an $L^{*}$ galaxy. Even if we suppose that the bulk of the dark matter is associated with very low surface brightness galaxies in clusters, the spatial distribution of these galaxies is required to be fine-tuned such that these effects do not show up in the shear field in the any of the three regions. In summary, the principal sources of uncertainty in the above analysis are (1) shot noise-we are inherently limited by the finite number of sources sampled within a few tidal radii of each cluster galaxy; (2) the spread in the intrinsic ellipticity distribution of the source population; (3) observational errors arising from uncertainties in the measurement of ellipticities from the images for the faintest objects; and (4) contamination by foreground galaxies mistaken as background.

The shot noise is clearly the most significant source of error, accounting for up to $\sim 50 \%$; followed by the width of the intrinsic ellipticity distribution that contributes $\sim 20 \%$, and the other sources together contribute $\sim 30 \%$ (Natarajan et al. 2007). This inventory of errors suggests that the optimal future strategy for such analyses is to go significantly deeper and wider in terms of the field of view.

\section{RESULTS FROM GALAXY-GALAXY LENSING}

The fiducial mass of a dark matter subhalo hosting an $L^{*}$ early-type galaxy in the central region contained within an aperture of size $r_{t *}=45 \pm 5 \mathrm{kpc}$ is $M=6.3_{-2.0}^{+2.7} \times 10^{11} M_{\odot}$; in the transition region it increases to $M=1.3_{-0.6}^{+0.8} \times 10^{12} M_{\odot}$, and in the periphery it increases further to $M=3.7_{-1.1}^{+1.4} \times 10^{12} M_{\odot}$. All error bars represent $3 \sigma$ values. These values derived from

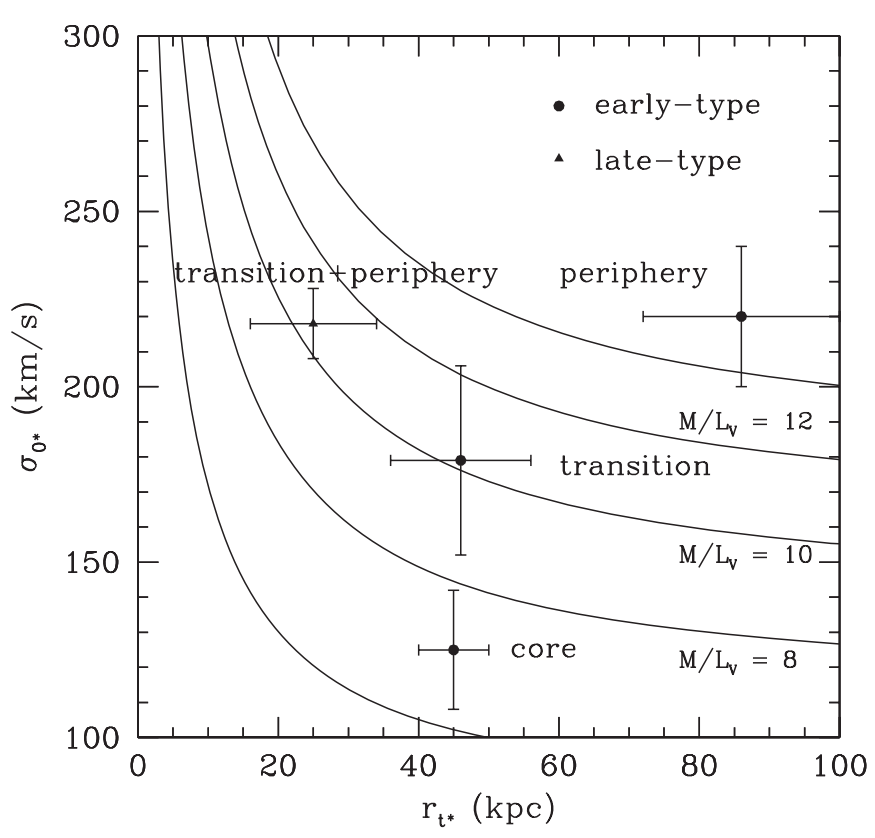

Figure 3. Fiducial value of the central velocity dispersion $\left(\sigma_{0 *}\right)$ and aperture radius $\left(r_{t *}\right)$ for an $L^{*}$ galaxy. These two parameters are chosen in the optimization for the PIEMD fit to the subhalos. The mass of a subhalo in the context of this model is proportional to $\sigma_{0 *}^{2} r_{t *}$. Overplotted are curves of constant mass-to-light ratio in the $V$-band with values $6,8,10,12$, and 14 (increasing from the bottom up). The solid circles are for subhalos associated with early-type galaxies and the solid triangle symbol is for the subhalo associated with late-type galaxies. The plotted error bars are $3 \sigma$ derived from the likelihood contours.

the likelihood analysis are shown in Figure 3. The increasing masses of the subhalos with cluster radius demonstrate that the subhalos that host $L^{*}$ galaxies in the inner regions (core and transition) are subject to more severe tidal truncation than those in the periphery. The mass of a typical subhalo that hosts an $L^{*}$ early-type galaxy increases with cluster-centric radius in concordance with theoretical expectations. The dark matter subhalo associated with a typical late-type galaxy in the transition and peripheral region is detected, with an aperture mass of $M=1.06_{-0.41}^{+0.52} \times 10^{12} M_{\odot}$ enclosed within a radius of $r_{t *}=25 \pm 5 \mathrm{kpc}$ (shown as the solid triangle in Figure 3). The total mass-to-light ratio for these fiducial subhalos can also be estimated. The constant total mass-to-light ratio curves overplotted in Figure 3 suggest that a typical subhalo hosting an $L^{*}$ early type has a $M / L_{V} \sim 7,10$, and 14 , respectively, in the three radial bins and a subhalo hosting an equivalent luminosity late-type galaxy has an $M / L_{V} \sim 10$. These values suggest that galaxies in clusters do possess individual dark matter subhalos that extend to well beyond the stellar component.

Utilizing the scaling with luminosity provided by the FaberJackson and Tully-Fisher relations, we derived the mass function of subhalos within each bin (Figure 4). Clearly, the core region where the central density of the cluster is maximal is expected to be an extreme and violent environment for infalling galaxies. We interpret our results to be a consequence of the fact that galaxies in the inner bin are more tidally truncated as they likely formed earlier and have therefore had time for many more crossings through the dense cluster center.

These results are in very good agreement with theoretical predictions wherein galaxies in the inner region are expected to be violently tidally stripped of their dark matter content, while those in the periphery are unlikely to have had even a single passage through the cluster center and therefore be untouched 


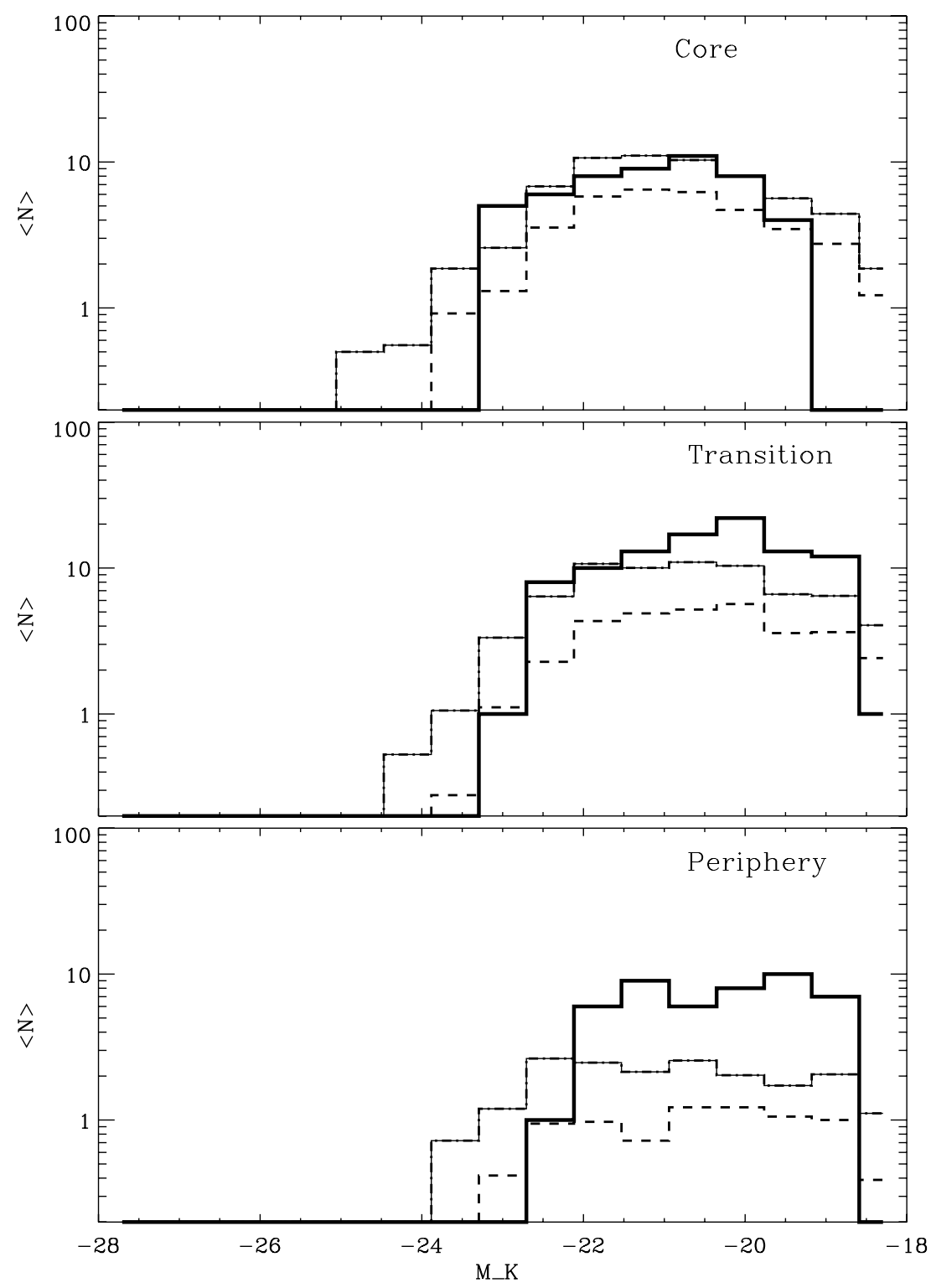

Figure 4. Luminosity function of the early-type galaxies in the three radial bins considered in this analysis. The value of $M_{K *}$ in the $K$-band is -21.37 . Note that the $y$-axis for the model galaxies is an averaged number $\langle N\rangle$ as the total number of selected early types is divided by 36 to take into account 12 clusters each with three independent projections. For the observed galaxies the $y$-axis denotes the raw number. In all panels, the thick solid histograms show the luminosity function of spectroscopically confirmed early-type members from the Cl0024+16 data set. The thin solid histograms are total luminosity function of early types in the simulations including Type 0 , Type 1 , and Type 2 galaxies. We separately show the luminosity function of Type 2 early-type galaxies as the dashed histograms. The fraction of Type 2 galaxies in the core is $58 \%$, in the transition region it is $50 \%$, and in the outer regions it is $46 \%$.

by tidal interactions. A simple analytic model (Merritt 1985) is used to predict the mass enclosed within the tidal radius ${ }^{14}$ as a function of cluster-centric distance, and is found to be consistent with our results (solid line in Figure 6). Our results are also consistent with the findings of Gao et al. (2004), who found that subhalos closer to the cluster center retain a smaller fraction of their dark matter. Furthermore, we are able to quantify the dark matter subhalo masses associated with late-type galaxies in $\mathrm{Cl} 0024+16$.

While the mean mass of a dark matter subhalo associated with an early-type cluster galaxy increases with cluster-centric distance out to $5 \mathrm{Mpc}$ and they trace the overall spatial distribution of the smooth mass components robustly. The subhalos associated with late-type galaxies do not contribute

14 The aperture radius $r_{t}$ that we infer from the lensing analysis is a proxy for the tidal radius of a dark matter subhalo. significantly to the total subhalo mass function at any radius. In fact, it appears that the host subhalos of late types do not trace the total dark matter distribution in clusters. We infer that the mass within $5 \mathrm{Mpc}$ in $\mathrm{Cl} 0024+16$ is distributed as follows: $\sim 70 \%$ of the total mass of the cluster is smoothly distributed, the subhalos associated with early-type galaxies contribute $\gtrsim 20 \%$, and subhalos hosting late-type galaxies account for the remaining $<10 \%$.

\subsection{Comparison with N-Body Simulations}

In this section, we compare the lensing results discussed above with the results from the Millennium Simulation (Springel et al. 2005). The simulation follows $N=2160^{3}$ particles in a box of size $500 h^{-1} \mathrm{Mpc}$ on a side, with a particle mass of $8.6 \times 10^{8} h^{-1} M_{\odot}$ (yielding several hundred particles per subhalo), and with a spatial resolution of $5 h^{-1} \mathrm{kpc}$. For each 
snapshot of the simulation (in total 64), substructures within dark matter halos have been identified using the algorithm SUBFIND (Springel et al. 2001). We refer to the original paper for more details on the algorithm. Further details of the determination of subhalo masses and the biases therein are discussed in Natarajan et al. (2007).

For our comparison with $\mathrm{Cl} 0024+16$, we have selected all cluster halos with $M_{200} \geqslant 8 \times 10^{14} M_{\odot}$ from the simulation box at $z \sim 0.4$. A total of 12 such cluster scale halos are found. We then use the publicly ${ }^{15}$ available results from the semianalytic model described in De Lucia \& Blaizot (2007) to select all galaxies in boxes of $10 h^{-1} \mathrm{Mpc}$ on a side and centered on the selected halos. We note that the following nomenclature is used for galaxies in the adopted semianalytic model: each FOF group hosts a "central galaxy" (Type 0) that is located at the position of the most bound particle of the main halo. All other galaxies attached to dark matter subhalos are labeled as Type 1 and located at the positions of the most bound particle of the parent dark matter substructure. Tidal truncation and stripping can disrupt the substructure down to the resolution limit of the simulation. A galaxy that is no longer identified with a dark matter subhalo is labeled as Type 2, and it is assumed not to be affected by processes that reduce the mass of its parent subhalo. The positions of Type 2 galaxies are tracked using the position of the most bound particle of the subhalo before it was disrupted.

We then select all galaxies brighter than $M_{K}=-18.3$ (this corresponds to all galaxies brighter than $1 / 20 * L^{*}$, as $\left.M_{K *}=-21.37\right)$ and classify as early types those with $\Delta M=M_{B}-M_{\text {bulge }}<0.4$, where $M_{B}$ is the $B$-band rest-frame magnitude and $M_{\text {bulge }}$ is the $B$-band rest-frame magnitude of the bulge (Simien \& de Vaucouleurs 1986). For each simulated cluster, we consider the same three radial bins used for our lensing analysis and stack the results for the projections along the $x$-, $y$-, and $z$-axes. This is done to mimic as best the projected distances that we employ in our lensing analysis in the three radial bins. In addition, we only consider galaxies within 1 $\mathrm{Mpc}$ (in the redshift direction) from the cluster center along the line of sight. This choice is motivated by the width of the measured velocity dispersion histogram in $\mathrm{Cl} \mathrm{0024+16}$ and therefore reduces contamination from unassociated structures. The inventory is as follows: core region-the models predict a total of $\sim 74$ early types that make the selection cut of which 42 are Type 2 galaxies and 32 are Type 0 and Type 1's; transition region-the models predict a total of 83 early-type galaxies that make the selection cut of which 41 are Type 2 galaxies and 42 are Type 0 and Type 1 's; outer region-the models predict a total of 22 early types that make the selection cut of which 10 are Type 2 galaxies and 12 are Type 0 and Type 1 's. In contrast, the selection from the observational data of $\mathrm{Cl} 0024+16$ yields the following numbers for spectroscopically confirmed early types with equivalent selection criteria: core region-51 early types; transition region-97 early types; outer region-47 early types.

In Figure 4, we plot the luminosity function of early-type galaxies in the three radial bins considered in this analysis from observations (thick, solid histograms) and the model (thin solid and dashed histograms). The thick solid histograms show the luminosity function of the spectroscopically confirmed early types in each bin. The thin, solid histograms show the total model luminosity function for equivalently selected early types (this includes Type 1's, Type 0's, and Type 2 galaxies). The dashed

\footnotetext{
15 A description of the publicly available catalogues, and a link to the database can be found at the following Web site:

http://mpa-garching.mpg.de/millennium/.
}

histograms show the luminosity function of Type 2 galaxies only. We note that in all regions the contribution by number of Type 2 galaxies is comparable to that of Type 0 and Type 1's. In the core region, $58 \%$ of all model early types are Type 2 's, in the transition region, $50 \%$ of all model early types are Type 2's, and in the outer region, $46 \%$ of all model early types are Type 2 galaxies.

It is clear from Figure 4 that the luminosity functions of the early-type galaxies in simulations agree rather well with the observed ones in all three bins. However, we note that the inability of the lensing analysis to accommodate/distinguish Type 2 galaxies that constitute roughly half the number of early types in the model will limit our analysis. This discrepancy in the total number of early types that are hosted in individual dark matter halos in the model versus the lensing analysis will reappear when we compare the masses of a typical subhalo that hosts an $L^{*}$ early-type galaxy.

In Figure 5, we compare the mass function of dark matter subhalos obtained from the galaxy-galaxy lensing analysis with that obtained from averaging the 12 massive clusters (each with three independent projections) in the Millennium Simulation. In the left-hand panel, we plot a direct comparison of the mass functions without taking into account the discrepancy in number between the observations and the simulations. In the right-hand panel, we scale the observations to take into account the fraction of Type 2's versus Type 0 and 1 's in each radial bin. This is done by normalizing the observations to match the fraction of Type 0 's and Type 1 's. In order to make a sensible comparison, we focus on the right-hand panel of Figure 5. In the core region, the mass function from simulations agrees quite nicely with that determined using the galaxy-galaxy lensing analysis. It is notable that general shapes of the mass function are in very good agreement. The agreement between the shape of the mass functions in transition and outer regions is also good. ${ }^{16}$

In the inner region, we found in earlier work (Natarajan et al. 2007) that the masses from the simulation tend to be underestimated by a factor 2 or so. This offset was found in our earlier analysis of the core regions in five clusters $(r<1$ $\mathrm{Mpc}$ ) reported in Natarajan et al. (2007). The origin of this offset in the core region has to do primarily with the systematics due the method employed to determine subhalo masses. The SUBFIND algorithm used to find dark matter substructures tends to underestimate their masses (for a more extensive discussion and diagnostic plots see Figure 3 in Natarajan et al. 2007) by a factor of 2 in the inner regions. Natarajan \& Springel (2005) and Natarajan et al. (2007) have also shown that the cluster-to-cluster variation for simulated halos is quite large. Therefore, we do not correct for this bias in the current analysis of $\mathrm{Cl} 0024+16$.

We note here that the subhalo mass function available from the simulations and the lensing technique probe a comparable mass range suggesting that our early-type galaxy selections have been equivalent. In the periphery, subhalo masses derived from lensing are of the order of few times $10^{13} M_{\odot}$, which is typical of group masses, suggestive of the presence of infalling groups. Tracking morphological types and their transformations

\footnotetext{
16 It is worth mentioning here that in Natarajan et al. (2007) Cl 0024+16 was the one outlier from the general good agreement. The lensing determined mass function for $\mathrm{Cl} 0024+16$ within $1 \mathrm{Mpc}$ was not in good agreement with the subhalo mass function derived from simulations. We attribute the current agreement of the mass functions in the core region $(r<0.6 \mathrm{Mpc})$ to the following two key factors (1) careful selection based on early-type cluster members to mimic the observations and (2) more careful classification into Type 1 and Type 2 galaxies and taking their relative numbers into account when computing the mass function.
} 

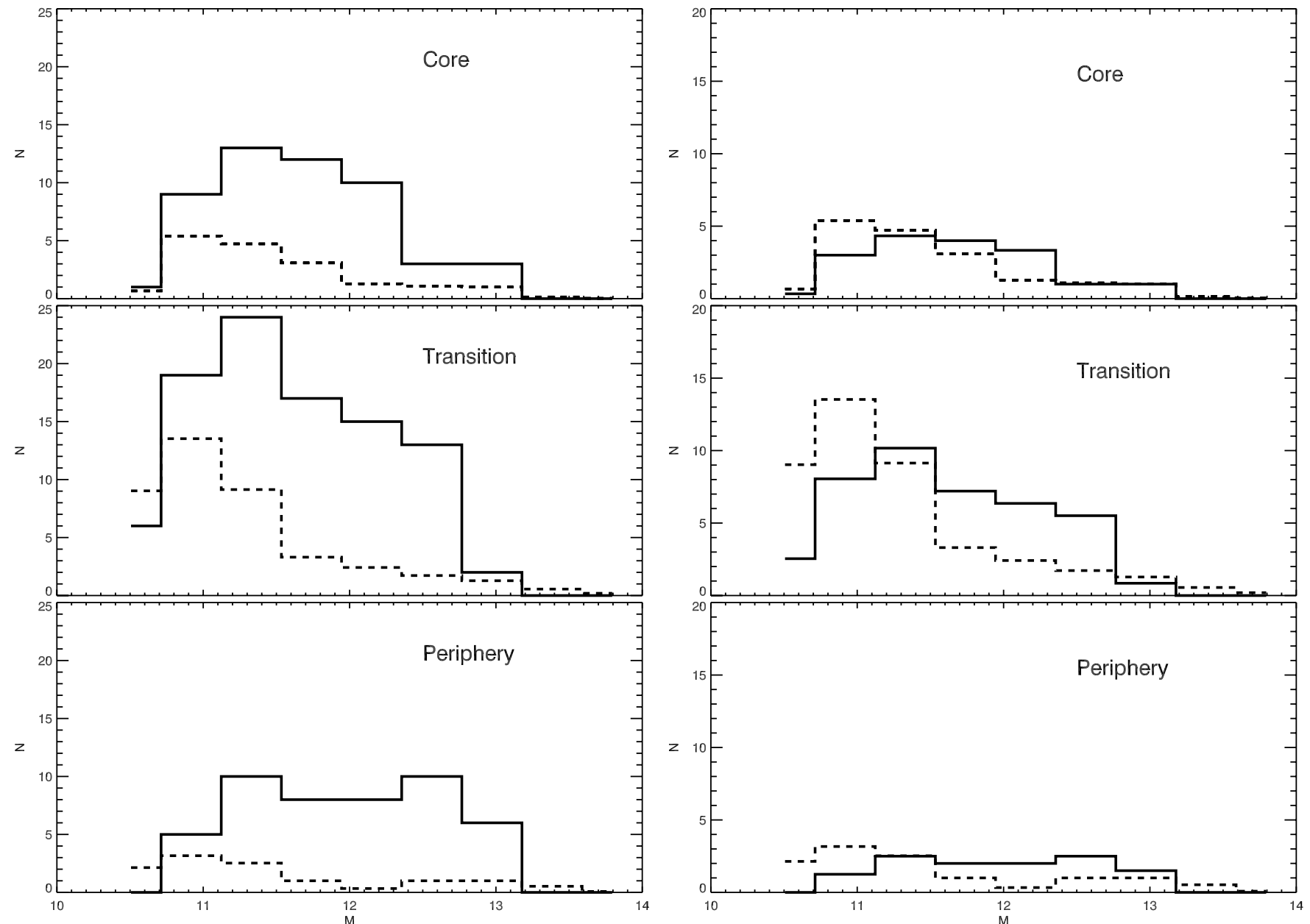

Figure 5. Comparison of the mass function determined from galaxy-galaxy lensing as a function of cluster-centric distance with that determined from simulated clusters in the Millennium Simulation. The solid histograms are the results from the lensing analysis and the dashed ones are from the Millennium Run. The raw mass function without any normalization or scaling is shown in the right-hand panel, whereas in the left-hand panel the lensing-derived mass function is normalized to compare with the model mass functions.

in this region Treu et al. (2003) also suggest the prevalence of infalling groups, in consonance with our lensing results. So we emphasize here, that in the outskirts of the Cl 0024+16, it appears that inferred subhalo masses correspond to group scale masses suggesting that these halos likely contain other fainter galaxies in addition to the bright, early type that we tag in this analysis.

In Figure 6, we plot the mass of a typical subhalo that hosts an early-type $L^{*}$ galaxy as a function of cluster-centric radius derived from galaxy-galaxy lensing and the simulations. The solid line in Figure 6 is the trend derived from a simple analytic model of tidal stripping of galaxies by an isothermal cluster proposed by Merritt (1985). The dashed line is the also the analytic model offset appropriately to compare with the simulation results. The model curve is nearly identical in slope to the best-fit line going through the simulation points. The radial trends are in very good agreement although there is an offset of a factor of $\sim 2.5$ in the mean value of the subhalo masses. It is likely that systematics in the lensing also contribute to this discrepancy. The efficiency of tidal stripping depends on the central density of the cluster. Writing this out explicitly, we have

$$
M_{\text {lens }} / M_{\text {sim }} \sim 2.5 \text {. }
$$

The offset in subhalo masses by a factor of $\sim 2.5$ suggests that the tidal stripping in the averaged simulated clusters is more ef- ficient than in $\mathrm{Cl} 0024+16$ as inferred from the lensing data. We note here that the values of $M_{200}$ for the 12 simulated clusters range from $\sim 8 \times 10^{14} M_{\odot}$ to $\sim 2 \times 10^{15} M_{\odot}$ and the best-fit parameters for $\mathrm{Cl} 0024+16$ from observations that consist of a superposition of $2 \mathrm{NFW}$ profiles with $M_{200} \sim 4 \times 10^{14} M_{\odot}$ and $M_{200} \sim 1.8 \times 10^{14} M_{\odot}$, could partially account for the discrepancy. The simulated ensemble does not reproduce the observed bimodal mass distribution in $\mathrm{Cl} 0024+16$ which has important dynamical consequences. No dynamical analog to $\mathrm{Cl} 0024+16$ was found in the Millennium Run at $z \sim 0.4$. Therefore it is not surprising that there is a discrepancy in the inferred mass for a dark matter subhalo hosting an $L^{*}$ galaxy. Note that if we correct the subhalo mass in the inner most bin by a factor of 2 as found in our earlier work, the agreement gets significantly better in the core region consistent with our earlier results (Natarajan et al. 2007). Regardless, there appears to be an offset despite overall agreement in the ensemble mass functions (as shown in the right-hand panel of Figure 5). Tidal stripping of dark matter appears to be more efficient in the simulations compared to estimates from the lensing data.

We note here that in the Millennium Simulation only the dark matter is followed dynamically but not the baryons. It has been recently argued that the adiabatic contraction of baryons in the inner regions of galaxies and clusters is likely to modify density profiles appreciably. Such modifications will impact 


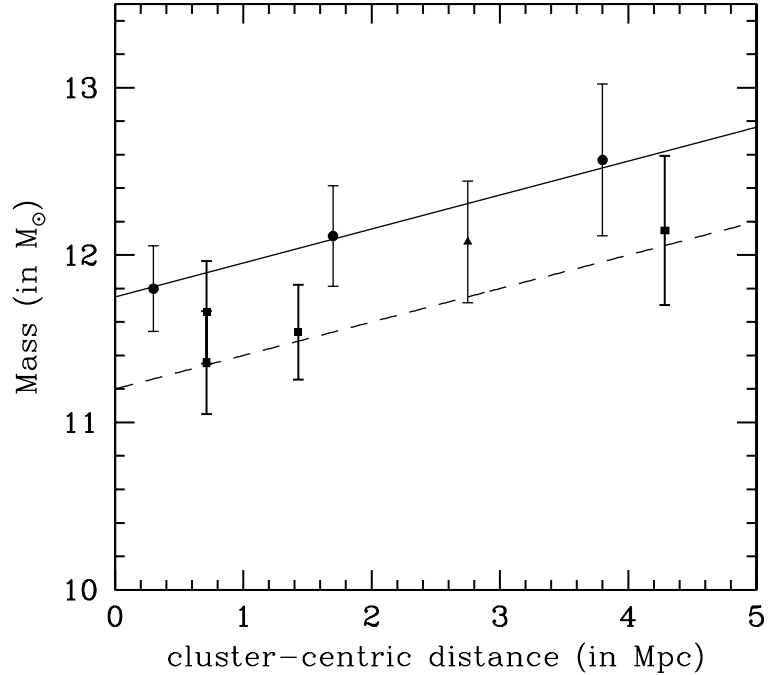

Figure 6. Variation of the mass of a dark matter subhalo that hosts an early-type $L^{*}$ galaxy as a function of cluster centric radius. The results from the likelihood analysis are used to derive the subhalo mass for the galaxy-galaxy lensing results and the counterparts are derived from the Millennium Simulation with an embedded semianalytic galaxy formation model. This enables selection of dark matter halos that host a single $L^{*}$ galaxy akin to our assumption in the lensing analysis. The solid circles are the data points from the galaxy-galaxy lensing analysis and the solid squares are from the Millennium Simulation. The upper solid square in the core region marks the value of the subhalo mass with correction by a factor of 2 as found in Natarajan et al. (2007). The solid triangle is the galaxy-galaxy lensing data point for the subhalo associated with a latetype $L^{*}$ galaxy. The radial trend derived from lensing is in very good agreement with simulations although there is an offset in the masses which is discussed further in the text.

the efficiency of tidal stripping in clusters. This claim has been made in numerical simulations that include gas cooling and prescriptions for star formation by Gnedin et al. (2004). Zappacosta et al. (2006) on the other hand claim using the case of the cluster Abell 2589 that adiabatic contraction is unimportant for the overall mass distribution of clusters. A recent study by Limousin et al. (2007b) that examines the tidal stripping of subhalos in numerical simulations and includes baryons finds a radial trend in the mass function that is in good agreement with the lensing-derived trend in $\mathrm{Cl} \mathrm{0024+16.}$

Meanwhile in lensing the systematic arises from the fact that we do not have measured redshifts for all background sources. While the mass calibration is most sensitive to the median redshift adopted for the background galaxies, biases are introduced if the median redshift is overestimated or underestimated.

We note that the galaxy-galaxy lensing technique is sensitive to the detection of subhalo masses above a threshold value that is determined by the quality of the observational lensing data. The selection made in the cluster luminosity function translates into a mass limit. The contribution of fainter early types (galaxies fainter than our selection limit) translates into lower mass subhalos due to the assumed luminosity scalings. As a consequence, subhalos with lower masses get included in the mass inventory as constituting the "smooth" component. The lensing-derived mass functions are therefore complete at the high-mass end but are typically incomplete at the lowmass end. The cutoff at the low-mass end is hence determined primarily by the depth of the observational data and the ability to measure shapes accurately for the faintest background sources. Since galaxy-galaxy lensing analysis in clusters is inherently statistical, its robustness is also limited by the ability to accurately pin down the smooth mass component, subtract it from the observed shear field and then stack the residuals to characterize the mass of a detectable dark matter subhalo. In the inner region while the constraints on the smooth component are tighter due to the presence of strong lensing features, the smooth component also tends to dominate the overall mass distribution, so subtracting it is challenging. In the outer regions while the smooth component is subdominant, there are fewer constraints and the overall value of the shear is significantly lower as well. These trade-offs cause a varying mass resolution for the lensing technique as a function of cluster-centric radius. However, since we assume scaling relations with luminosity, and use the cluster galaxy luminosity function to determine the mass function, the detectable limit of subhalo masses is set predominantly by the magnitude cut adopted for the selected early-type galaxies. Note that in our comparison with simulations we have restricted ourselves only to early types with measured spectroscopic redshifts. Since spectroscopic follow-up tends to be easier for brighter galaxies, once again our lensing-derived mass function is more complete at the high-mass end and is less so at the lowmass end. However, from the comparison of the mass functions we note that both methods lensing and the simulations are probing comparable subhalo mass ranges.

\section{DISCUSSION AND CONCLUSIONS}

Earlier work on galaxy-galaxy lensing in the field has identified a signal associated with massive halos around typical field galaxies, extending to beyond $100 \mathrm{kpc}$ (e.g., Brainerd et al. 1996; Ebbels et al. 1998; Hudson et al. 1998; Wilson et al. 2001; Hoekstra et al. 2005). In particular, Hoekstra et al. (2005) report the detection of finite truncation radii via weak lensing by galaxies based on $45.5 \mathrm{deg}^{2}$ of imaging data from the RedSequence Cluster Survey. Using a truncated isothermal sphere to model the mass in galaxy halos, they find a best-fit central velocity dispersion for an $L^{*}$ galaxy of $\sigma=136 \pm 5 \mathrm{~km} \mathrm{~s}^{-1}$ (68\% confidence limits) and a truncation radius of $185 \pm 30$ kpc. Galaxy-galaxy lensing results from the analysis of the Sloan Digital Sky Survey data (Sheldon et al. 2004; Guzik \& Seljak 2002; Mandelbaum et al. 2006) have contributed to a deeper understanding of the relation between mass and light. Similar analysis of galaxies in the cores of rich clusters suggests that the average mass-to-light ratio and spatial extents of the dark matter halos associated with morphologically classified early-type galaxies in these regions may differ from those of comparable luminosity field galaxies (Natarajan et al. 1998, 2002). We find that at a given luminosity, galaxies in clusters have more compact halo sizes and lower masses (by a factor of 2-5) compared to their field counterparts. The mass-to-light ratios inferred for cluster galaxies in the $V$ band are also lower than that of comparable luminosity field galaxies. This is a strong indication of the tidal stripping effect of the dense environment on the properties of dark matter halos. In recent work, using only strong lensing constraints in the inner regions of the Abell cluster A 1689 derived from images taken by the Advanced Camera for Surveys (ACS) aboard HST, Halkola \& Seitz (2007) also find independently that the subhalos of cluster galaxies are severely truncated compared to equivalent luminosity galaxies in the field.

The subhalo mass function represents an important prediction of hierarchical CDM structure formation models and has been subject of intense scrutiny since the "satellite crisis" was identified (Moore et al. 1999). This crisis refers to the fact that within a radius of $400 h^{-1} \mathrm{kpc}$, from the Milky Way, cosmological simulations of structure formation predict 
$\sim 50$ dark matter satellites with circular velocities in excess of $50 \mathrm{~km} \mathrm{~s}^{-1}$ and mass greater than $3 \times 10^{8} M_{\odot}$. This number is significantly higher than the dozen or so satellites actually detected around our Galaxy. Several explanations have been proposed to resolve this discrepancy. The missing satellites could, for example, be identified with the detected High Velocity Clouds (Kerr \& Sullivan 1969; Willman et al. 2002; Maller \& Bullock 2004). Warm or self-interacting dark matter could also selectively suppress power on the small scales, therefore reducing the predicted number of satellites. The leading hypothesis, however, remains that the solution to this problem lies in processes such as heating by a photo-ionizing background that preferentially suppresses star formation in small halos at early times. On the scale of galaxy clusters, many more dark matter structures are expected to be visible, thus making the comparison with expectation from numerical simulations less affected by uncertainties in the poorly understood physics of the galaxy formation. In earlier work, we showed that there is very good agreement between the lensing observations and the Millennium Run simulations in the inner $1 \mathrm{Mpc}$ or so for a sample of clusters (Natarajan et al. 2007). Now we are able to extend our analysis out to $5 \mathrm{Mpc}$ for $\mathrm{Cl} 0024+16$ due to the unique data set that is available. The diagnostic available for comparison here is the shape of the mass function in each of the three bins. We find that the overall shape of the subhalo mass functions derived from the two independent methods is in very good agreement out to beyond the virial radius. We find that the mass of a typical subhalo that hosts an $L^{*}$ early-type galaxy increases with cluster-centric radius in concordance with theoretical expectations. However, the estimates of the mass of a subhalo that hosts an $L^{*}$ galaxy derived from simulations is significantly lower than those derived from lensing observations. The origin of this discrepancy lies in the fact that tidal stripping appears to be more efficient in the simulations.

Due to the large area probed by this $\mathrm{Cl} 0024+16$ data set, we are also able to constrain the properties of dark matter subhalos associated with late-type galaxies that preferentially lie in the outer regions of the cluster (Treu et al. 2003). We report the first detection of the presence of a dark matter subhalo associated with late-type galaxies in $\mathrm{Cl} 0024+16$. While early-type galaxies appear to trace the overall mass distribution robustly, the subhalos associated with late-type galaxies do not contribute significantly to the total mass budget at any radius. In the cluster $\mathrm{Cl} 0024+16$ within $5 \mathrm{Mpc}$ we find the following contributions to the total mass: $\sim 70 \%$ of the total mass of the cluster is smoothly distributed, the subhalos associated with early-type galaxies contribute $\gtrsim 20 \%$, and subhalos hosting late-type galaxies account for the remaining $<10 \%$.

The mass resolution of our technique varies slightly with cluster-centric distance owing to the nature of observational constraints that dominate the likelihood optimization. While the strong lensing constraints in the core are the most stringent and drive the fit in the inner regions, the anisotropy in the shear field is statistically harder to recover. As we progressively step out in radius away from the cluster center, the shear of the large-scale smooth component drops, and that of the individual subhalos dominates but the overall summed shear signal is significantly lower than in the inner regions. The current analysis is primarily limited by the quality of the available data. Data sets from the $A C S$ will allow mass modeling of lensing clusters at even higher resolutions providing increasing accuracy enabling better mapping of the lower-mass end of the mass functions of substructure. However, as described above a vast complement of ground-based observations are also needed for this kind of comprehensive analysis which is extremely time consuming. Ground-based data provided many important constraints, for instance, the large number of measured central velocity dispersions for cluster galaxies (Moran et al. 2007) was used as priors in modeling the perturbing subhalos that made the optimization more efficient.

Below we summarize the key results on comparison with simulations, where we mimicked the selection process adopted for the observational data of $\mathrm{Cl} 0024+16$. Dividing the simulated clusters drawn from the Millennium Run into three equivalent radial bins as the observational data, we were able to estimate (1) the mass function in each bin and (2) the subhalo masses that host $L^{*}$ early-type galaxies. The shapes of the lensing-derived mass functions are in reasonable agreement with those derived from simulations when we normalize the lensing results to the those of the total number of model Type 1's and Type 2's.

Our results provide strong support for the tidal stripping hypothesis. We also find evidence for the variation in the efficiency of tidal stripping with cluster-centric radius and morphological type. We conclude that dark matter in clusters is assembled by the incorporation of infalling subhalos that are progressively stripped during their journey through the cluster. The finding of kinematically disturbed features in the cluster galaxy population by Moran et al. (2007) corroborates our conclusion. We have significantly improved on previous ground-based studies as space-based data afford greater accuracy in shape measurements. Future space-based surveys coupled with ground-based spectroscopic follow-up will provide an unprecedented opportunity to follow the cluster assembly process.

Pranjal Trivedi is thanked for help during the early stages of this project. Gabriella De Lucia is gratefully acknowledged for her help in extracting catalogs from the Millennium Simulation, in the analysis of simulated data and thoughtful comments on the draft. Help from Volker Springel is acknowledged for clarifications regarding subhalo extraction from the Millennium Simulation. Some of this work was conducted using support from the grant HST-GO-09722.06-A provided by NASA through a grant from Space Telescope Science Institute, which is operated, by the Association of Universities for Research in Astronomy, Incorporated, under NASA contract NAS5-26555. This paper is based on the observations made with the NASA/ESA Hubble Space Telescope, which is operated by the Association of Universities for Research in Astronomy, Inc., under NASA contract NAS 5-26555. IRS acknowledges support from the Royal Society. ML acknowledges support from the Dark Cosmology Centre funded by the Danish National Research Foundation. The Millennium Simulation databases used in this paper and the web application providing online access to them were constructed as part of the activities of the German Astrophysical Virtual Observatory.

\section{APPENDIX}

\section{THE INTRINSIC SHAPE DISTRIBUTION OF BACKGROUND GALAXIES}

As in all lensing work, it is assumed here as well, that the intrinsic or undistorted distribution of shapes of galaxies is a known quantity. This distribution is obtained from shape measurements taken from deep images of blank field surveys. Previous analysis of deep survey data such as the MDS fields showed that the ellipticity distribution of sources is a strong 
function of the sizes of individual galaxies as well as their magnitude (Kneib et al. 1996). For the purposes of our modeling, the intrinsic ellipticities for background galaxies are assigned in concordance with an ellipticity distribution $\left.p_{(} \tau_{S}\right)$ where the shape parameter $\tau$ is defined as $\tau=\left(a^{2}-b^{2}\right) /(2 a b)$ derived from the observed ellipticities of the CFHT12k data (see Limousin et al. 2007a for details):

$$
p\left(\tau_{S}\right)=\tau_{S} \exp \left(-\left(\frac{\tau_{S}}{\delta}\right)^{v}\right) ; v=1.15, \delta=0.25 .
$$

This distribution includes accurately measured shapes of galaxies of all morphological types. In the likelihood analysis this distribution $p\left(\tau_{S}\right)$ is the assumed prior, which is used to compare with the observed shapes once the effects of the assumed mass model are removed from the background images. We note here that the exact shape of the ellipticity distribution, i.e., the functional form and the value of $\delta$ and $v$ do not change the results but alter the confidence levels we obtain. The width of the intrinsic ellipticity distribution, on the other hand, is the fundamental limiting factor in the accuracy of all lensing measurements including this work.

\section{THE REDSHIFT DISTRIBUTION OF BACKGROUND GALAXIES}

While the shapes of lensed background galaxies can be measured directly and reliably by extracting the second moment of the light distribution, in general, the precise redshift for each weakly object is in fact unknown and therefore needs to be assumed. Using multiwaveband data from surveys such as COMBO-17 photometric redshift estimates can be obtained for every background object. Typically the redshift distribution of background galaxies is modeled as a function of observed magnitude $P(z, m)$. We have used data from the high-redshift survey VIMOS VLT Deep Survey as well as recent CFHT12k $R$-band data to define the number counts of galaxies, and the HDF prescription for the mean redshift per magnitude bin, and find that the simple parameterization of the redshift distribution used by Brainerd et al. (1996) still provides a good description to the data. We also used CFHT $K$-band photometry to derive photometric redshifts and an $N(z)$ for all the background sources in $\mathrm{Cl}$ 0024+16 (Kneib et al. 2003; Smith et al. 2005) but found that drawing instead from the distribution below allowed us to go deeper to the magnitude limits required. We used the derived photometric redshifts for sources with reliable estimates and for the rest we drew from the distribution below. For the normalized redshift distribution at a given magnitude $m$ (in the $I_{814}$ band) we have

$$
\left.N(z)\right|_{m}=\frac{\beta\left(\frac{z^{2}}{z_{0}^{2}}\right) \exp \left(-\left(\frac{z}{z_{0}}\right)^{\beta}\right)}{\Gamma\left(\frac{3}{\beta}\right) z_{0}}
$$

where $\beta=1.5$ and

$$
z_{0}=0.7\left[z_{\text {median }}+\frac{d z_{\text {median }}}{d m_{R}}\left(m_{R}-m_{R 0}\right)\right],
$$

$z_{\text {median }}$ being the median redshift, $d z_{\text {median }} / d m_{R}$ the change in median redshift with say the $R$-band magnitude, $m_{R}$.

The final results on the aperture mass presented here are also sensitive primarily only to the choice of the median redshift of the distribution rather than the individual assigned values. Since the median redshift of the distribution we adopt here is similar to that of COSMOS survey, our results would be robust.

\section{REFERENCES}

Abadi, M., Moore, B., \& Bower, R. 1999, MNRAS, 308, 947

Balogh, M., Navarro, J., \& Morris, S. 2000, ApJ, 540, 113

Bardeau, S., Soucail, G., Kneib, J.-P., Czoske, O., Ebeling, H., Hudelot, P., Smail, I., \& Smith, G. P. 2007, A\&A, 470, 449

Blandford, R., \& Narayan, R. 1992, ARA\&A, 30, 311

Bradac, M., et al. 2006, ApJ, 652, 937

Brainerd, T., Blandford, R. D., \& Smail, I. R. 1996, ApJ, 466, 623

Bridle, S., Kneib, J.-P., Bardeau, S., \& Gull, S. F. 2002, in Shapes of Galaxies and their Dark Matter Halos, ed. P. Natarajan (New York: World Scientific), 38

Broadhurst, T., Huang, X., Frye, B., \& Ellis, R. S. 2000, ApJ, 534, L15

Broadhurst, T., Takada, M., Umetsu, K., Kong, X., Arimoto, N., Chiba, M., \& Futamase, T. 2005, ApJ, 619, L143

Clowe, D., et al. 2006, A\&A, 451, 395

Cortese, L., et al. 2008, MNRAS, 383, 1519

Czoske, O., Kneib, J.-P., Soucail, G., Bridges, T. J., Mellier, Y., \& Cuillandre, J.-C. 2001, A\&A, 372, 391

Czoske, O., Moore, B., Kneib, J.-P., \& Soucail, G. 2002, A\&A, 386, 31

De Lucia, G., \& Blaizot, J. 2007, MNRAS, 375, 2

De Lucia, G., Kauffmann, G., Springel, V., White, S. D. M., Lanzoni, B., \& Stoehr, F. 2004, MNRAS, 348, 333

Dressler, A. 1980, ApJ, 236, 351

Ebbels, T., Ellis, R. S., Kneib, J.-P., Leborgne, J.-P., Pello, R., Smail, I. R., \& Sanahuja, B. 1998, MNRAS, 295, 75

Fasano, G., Poggianti, B., Couch, W. J., Bettoni, D., Kjaergaard, P., \& Moles, M. 2000, ApJ, 542, 673

Gao, L., White, S. D. M., Jenkins, A., Stoehr, F., \& Springel, V. 2004, MNRAS, 355,819

Gavazzi, R., Fort, B., Mellier, Y., Pello, R., \& Dantel-Fort, M. 2003, A\&A, 403, 11

Gavazzi, R., Mellier, Y., Fort, B., Cuillandre, J.-C., \& Dantel-Fort, M. 2004, A\&A, 422, 407

Geiger, B., \& Schneider, P. 1998, MNRAS, 295, 497

Ghigna, S., Moore, B., Governato, F., Lake, G., Quinn, T., \& Stadel, J. 1998, MNRAS, 300, 146

Gnedin, O., Kravtsov, A., Klypin, A., \& Nagai, D. 2004, ApJ, 616, 16

Guzik, J., \& Seljak, U. 2002, MNRAS, 335, 311

Halkola, A., \& Seitz, S. 2007, ApJ, 656, 739

Halkola, A., Seitz, S., \& Pannella, M. 2007, ApJ, 656, 739

Hoekstra, H., Hsieh, B. C., Yee, H. K. C., Lin, H., \& Gladders, M. 2005, ApJ, 635,73

Hudson, M., Gwyn, S. D. J., Dahle, H., \& Kaiser, N. 1998, ApJ, 503, 531

Jullo, E., Kneib, J-P., Limousin, M., Eliasdottir, A., Marshall, P., \& Verdugo, T. 2007, NJ Phys., 9, 447

Kassiola, A., \& Kovner, I. 1993, ApJ, 417, 474

Kerr, F., \& Sullivan, W. 1969, ApJ, 158, 115

Kneib, J.-P. 1993, PhD thesis, Univ. Paul Sabatier, Toulouse

Kneib, J.-P., Ellis, R. S., Smail, I. R., Couch, W. J., \& Sharples, R. M. 1996, ApJ, 471,643

Kneib, J.-P., et al. 2003, ApJ, 598, 804

Kuijken, K. 1999, A\&A, 352, 355

Limousin, M., Kneib, J-P., Bardeau, S., Natarajan, P., Czoske, O., Smail, I., Ebeling, H., \& Smith, G. P. 2007a, A\&A, 461, 881

Limousin, M., Kneib, J-P., \& Natarajan, P. 2005, MNRAS, 356, 309

Limousin, M., Sommer-Larsen, J., Natarajan, P., \& Milvang-Jensen, B. 2007b, A\&A, 461, 881

Limousin, M., et al. 2009, ApJ, in press (arXiv:astro-ph/0612165)

Maller, A., \& Bullock, J. 2004, MNRAS, 355, 694

Mandelbaum, R., Seljak, U., Kauffmann, G., Hirata, C, \& Brinkmann, J. 2006, MNRAS, 368, 715

Mellier, Y. 2002, Space Sci. Rev., 100, 73

Merritt, D. 1983, ApJ, 264, 24

Merritt, D. 1985, ApJ, 289, 18

Moore, B., Katz, N., Lake, G., Dressler, A., \& Oemler, A. 1996, Nature, 379, 613

Moore, B., Lake, G., Quinn, T., \& Stadel, J. 1999, MNRAS, 304, 465

Moran, S. M., Ellis, R. S., Treu, T., Smail, I. R., Dressler, A., Coil, A., \& Smith, G. P. 2005 , ApJ, 633, 32

Moran, S. M., Ellis, R. S., Treu, T., Smith, G. P., Rich, R. M., \& Smail, I. 2007, ApJ, 671, 1503

Natarajan, P., De Lucia, G., \& Springel, V. 2007, MNRAS, 376, 180

Natarajan, P., \& Kneib, J.-P. 1996, MNRAS, 293, 1031

Natarajan, P. \& Kneib, J.-P. 1997, MNRAS, 287, 833

Natarajan, P., Kneib, J.-P., Smail, I., \& Ellis, R. 2004, arXiv:astro-ph/0411426

Natarajan, P., Kneib, J.-P., \& Smail, I. R. 2002, ApJ, 580, L10 
Natarajan, P., Kneib, J.-P., Smail, I. R., \& Ellis, R. S. 1998, ApJ, 499, 600

Natarajan, P., \& Springel, V. 2005, ApJ, 617, L13

Navarro, J. F., Frenk, C. S., \& White, S. D. M. 1997, ApJ, 490, 493

Navarro, J. F., et al. 2004, MNRAS, 349, 1039

Okamoto, T., \& Habe, A. 1999, ApJ, 516, 591

Sand, D., Treu, T., Smith, G., \& Ellis, R. S. 2004, ApJ, 604, 88

Schneider, P., Ehlers, J., \& Falco, E. 1992, in Gravitational Lenses (Berlin: Springer)

Schneider, P., \& Rix, H.-W. 1997, ApJ, 474, 25

Sheldon, E., et al. 2004, A\&A, 127, 2544

Simien, F., \& de Vaucouleurs, G. 1986, ApJ, 302, 564

Smail, I. R., Dressler, A., Kneib, J.-P., Ellis, R. S., Couch, W. J., Sharples, R. M., \& Oemler, A. 1996, ApJ, 469, 508

Smith, G. P., et al. 2005, ApJ, 620, 78
Springel, V., White, S. D. M., Tormen, G., \& Kauffmann, G. 2001, ApJ, 328, 726

Springel, V., et al. 2005, Nature, 435, 629

Tormen, G., Diaferio, A., \& Syer, D. 1998, MNRAS, 299, 728

Treu, T., Ellis, R. S., Kneib, J.-P., Dressler, A., Smail, I. R., Czoske, O., \& Oemler, A. 2003, ApJ, 591, 53

Tyson, J. A., Kochanski, G. P., \& dell'Antonio, I. P. 1998, ApJ, 498, L101

Willman, B., Dalcanton, J., Ivezic, Z., Schneider, D., \& York, D. G. 2002, AJ, 124,2600

Wilson, G., Kaiser, N., \& Luppino, G. 2001, ApJ, 555, 572

Wittman, D., Dell'Antonio, I., Hughes, J. P., Margoniner, V., Tyson, J. A., Cohen, J., \& Norman, D. 2006, ApJ, 643, 128

Zappacosta, L., Buote, D., Gastaldello, F., Humphrey, P., Bullock, J., Brighenti, F., \& Mathews, W. 2006, ApJ, 650, 777 